\title{
Confluent Macular Lepromatous Leprosy
}

\author{
S. G. BROWNE, O.B.E., M.D., F.R.C.P., F.R.C.S., D.T.M. \\ Leprosy Service Research Unit, Uzuakoli, Eastern Nigeria
}

Under the title 'masked lepromatous leprosy', Davey (r942) described the clinical features of a variety of lepromatous leprosy in which confluent macules occupied virtually the whole of the skin, sparing a few areas. The patient, healthy and symptomless, passed for one of the lighter-hued members of society: the widespread uniform hypopigmentation was not apparent until he was completely stripped for examination, when normally pigmented skin was seen to be restricted to the pelvic girdle.

Owing to the interplay of diverse factors (such as the changing pattern of leprosy in Eastern Nigeria, the non-recognition of early macular leprosy, and the diagnostically confusing prevalence of macular mycotic lesions), confluent macular lepromatous leprosy of the type to which Davey (1942) drew attention, now accounts for rather more than io per cent of all new cases of lepromatous leprosy diagnosed in Eastern Nigeria. Frequently, the macules are not noticed, or, if noticed, they are not recognized for what they are, and it is only when lepromatous nodules appear that the thoughts of the patient or his family turn to leprosy.

It may therefore be salutary to record the clinical course and features and the bacteriological findings in a series of 13 such patients recently diagnosed in the Research Unit, Uzuakoli. Many other patients with essentially similar clinical manifestations have been studied during the past few months at the other Leprosy Settlements in Eastern Nigeria, viz., Oji River, Abakaliki, Ogoja, Uburu, Itu and Ekpene Obom.

\section{Mode of presentation}

Every patient in this series came for diagnosis and treatment because typical lepromatous nodules had recently appeared on the face or ears. (Nodular leprosy arising de novo and without macular or other signs is an uncommon presentation among the deeply-pigmented peoples of West Africa). None of the patients complained of any symptoms referable to the skin or peripheral nerves, and all denied any change in skin colouring. They would pass without remark in a crowd of typical Nigerians, who represent a very wide range of skin hue, from light yellow-brown to very dark brownblack.

\section{Clinical features}

When the patients stripped completely, it was evident that (apart from the hairy scalp) the only areas of skin in which the pigmentation was unchanged and the fine architecture undisturbed were to be found in the inguinal region, the lumbar region and the axillae. The superficial extent of this normally pigmented skin varied somewhat, but the basic pattern remained. In the most marked instances, it was reduced to a narrow band about an inch wide just above the ilio-inguinal (Poupart's) ligament, passing over the anterior superior iliac spine, and broadening out slightly above the iliac crest to meet the contralateral band in the midline. The limits of the bordering hypopigmented areas were indistinct, and the width of skin between the obviously normal and the definitely hypopigmented might be as great as an inch. The neck skin is sometimes spared.

One source of difficulty was the individual differences in the skin texture and natural depth of skin pigmentation between the buttocks, and the region just above the iliac crests, and superiorly. A similar state of affairs existed in the neighbourhood of the axillae, though here the condition was complicated by the presence of hair, constant perspiration, and frequently by mycotic and bacterial infection of both hair and skin.

There was very slight tactile impairment in the hypopigmented skin, compared with adjacent normal skin; sweating was unaffected, except in the later stages.

\section{Natural history}

By observation of the evolution of these macular lesions, it is possible to piece together a typical 
history. Scattered small ill-defined macules, very slightly hypopigmented, first appear usually in the skin of the scapular region, sometimes (in the young and the adolescent) following hazy patches or pre-lepromatous macules. Further crops of similar macules may appear on the back, abdomen, chest, limbs and face. At this stage, an irregular hypopigmented mottling in which the normal skin is represented by slender, darker streaks is the only abnormality visible. The macules extend centrifugally until they coalesce, but further pigment loss does not occur.

When the coalescence has proceeded down to the level of the umbilicus and up to the level of the mid-thigh, discrete macules of precisely similar appearance and degree of hypopigmentation may still be distinguished between the limits of the confluent macules and the areas of apparently normal skin. These discrete macules at length also coalesce.

The process, from start to finish may take from three to five years. It may stop at the macular stage, or progress towards the nodular.

Meanwhile, other features of lepromatous leprosy may be developing, till the full characteristic picture is produced. The eyebrows become thinned, especially the outer third or half, and are eventually completely lost. The peripheral nerve trunks become enlarged and tender at the usual sites, usually from about the third or fourth year onwards.

The skin becomes generally thickened, and less mobile than normal; sometimes the cutis is irregularly lumpy and feels bound down in places to the deep fascia. The central part of the face takes on a greasy appearance.

When acute exacerbation ensues, irregular infiltration and aggregations of pinkish or orange papular masses, together with lepromatous nodules, appear indiscriminately on the considerable areas of skin already the seat of the confluent hypopigmented macules. No acute lesions arise in the areas of apparently normal skin.

The series of I 3 patients now reported (I 2 males and one female, whose ages ranged from I 3 to 40), may be considered typical of some scores at all stages studied, the common feature being the existence of a generalized confluent hypopigmentation of the skin, regularly sparing the three regions specified.

\section{Clinical findings}

On the first examination, the extent of the area of apparently normal skin varied necessarily from patient to patient, depending on the actual point in the natural history of the condition when the patient came for diagnosis. In some patients, a few discrete macules were still present around the areas of apparently normal skin.

In 12 patients, the peripheral nerves showed some degree of enlargement. The ulnar nerves were affected precociously, and showed greater departures from the normal than the external popliteals or the posterior tibials. Gross en." largement coupled with hardness of the peripheral nerves at the sites of predilection was not found.

In three patients, there was already some symmetrical thinning of the external third of the eyebrows, and in one patient almost complete madarosis.

One patient had experienced an acute exacerbation in the confluent macular lesions just before admission. Sudden swelling and shininess of the helices of the ears brought another for diagnosis.

Atrophy of the pulp of the little fingers was seen in one patient, together with obliteration of the ridge pattern; and grosser wastage of the hypothenar eminence was present in another. One patient had discrete lepromatous lesions deep in the palmar skin. Early bilateral gynaecomastia was noted once.

Thus, all the patients in this series had at least one clinical feature of more advanced lepromatous leprosy, in addition to the widespread confluent macular rash. In another series, the patients came for diagnosis at an earlier stage in the natural history of the disease, corresponding very closely to Davey's ( I942) patient.

One patient has initially a skin of lighter hue than the average. $\mathrm{He}$, too, lost pigment in the same situations as his fellows.

\section{Bacteriological findings}

Smears taken from the usual eight sites (four from the skin, two from the ear-lobes, and one from each side of the nasal septal mucosa) all contained numerous Myco. leprae and globi. The average Bacterial Index (B.I.) for the I 3 patients was 3.I7 (maximum: 4.o, on Dharmendra's notation); in seven the B.I. was greater than 
3.0 , and in six greater than 2.0. The average percentage of 'solid rods' (Morphological Index) was 68 .

Interest centres around the smears taken from the three areas of apparently normal skin as compared with those from the obviously hypopigmented skin.

In Io patients, smears from the inguinal region contained bacilli, and in seven of these globi were found.

In all i3 patients, smears from the lumbar region contained bacilli, sometimes very numerous, with globi in all but two.

In I I patients, bacilli were found in the axillary skin, with globi in seven.

The morphology of the bacilli corresponded to that found in the adjacent hypopigmented skin.

The concentration of bacilli in these three regions was lower than that in the adjacent skin. Both solid rods and degenerate forms tended to disappear earlier from these regions than from the more heavily infected skin.

It is to be noted that the concentration of Myco. leprae (including globi) was consistently lower than that found in the skin between the macules at an earlier stage in the natural history of the disease. In the latter case, the concentration of bacilli and of globi, and the proportion of solid rods are usually comparable with results obtained in the adjacent obviously affected skin. The loss of pigment within the macular areas appears not to depend only or directly on the actual presence of lepromatous granuloma in the dermis.

\section{Immunology}

The lepromin test, both Fernandez and Mitsuda, was completely negative in all patients.

\section{Histology}

Discrete groups of heavily bacillated lepra cells were present in the dermis. The appearances were typical of early lepromatous leprosy.
Dis CUSSION

In this series of 13 patients, the confluent lepromatous macules had been unnoticed, and it was only the appearance of some additional feature that brought the patient for diagnosis. In other and more fortunate cases, leprosy has been suspected before the appearance of such features, when the symptomless, coalescent macules have been the only manifestation of the disease.

Various explanations have been offered to account for the sparing of these three areas of skin from the hypopigmentary effect of the lepromatous process: temperature of the epidermis and the dermis, sweating, skin $\mathrm{pH}$, influence of clothing, pressure, apocene secretions, etc. but none of these factors is common to all three sites.

The clinical and epidemiological importance of confluent macular lepromatous leprosy needs no emphasis. From the standpoint of the individual patient and that of the community, the condition should be recognized and diagnosed and treated before the onset of irreversible nerve damage.

\section{S U M M A R Y}

A series of I 3 patients is reported, in whom a generalized hypopigmentation was for some time the only evidence of leprosy, until other indubitable manifestations of lepromatous disease appeared. The hypopigmentation resulted from the confluence of macular areas, which regularly spared the inguinal region, a band of skin in the lumbar region, and the axillae. These areas of apparently normal skin usually harboured Myco. leprae and globi, though not in the same concentration as in neighbouring hypopigmented skin.

\section{A CKNOWLEDGEMENT}

My thanks are due to Dr S. O. Egwuatu, Chief Medical Officer, Ministry of Health, Eastern Nigeria, for permission to publish this article.

REFERENCE

DAVEy, T. F. (1942). Leprosy Rev., 13, 3-5. 